\title{
A model combining TNM stage and tumor size shows utility in predicting recurrence among patients with hepatocellular carcinoma after resection
}

This article was published in the following Dove Press journal: Cancer Management and Research

\author{
Yu Zhang ${ }^{1,2, *}$ \\ Shu-wei Chen $2,3, *$ \\ Li-li Liu ${ }^{1,2}$ \\ Xia Yang ${ }^{1,2}$ \\ Shao-hang Cai ${ }^{1,2}$ \\ Jing-ping Yun ${ }^{1,2}$
}

'Department of Pathology, Sun Yat-sen University Cancer Center, Guangzhou, People's Republic of China; ${ }^{2}$ Sun Yat-sen University Cancer Center, State Key Laboratory of Oncology in South China, Collaborative Innovation Center for Cancer Medicine,

Guangzhou, People's Republic of China; ${ }^{3}$ Department of Head and Neck Surgery, Sun Yat-sen University Cancer Center, Guangzhou, People's Republic of China

*These authors contributed equally to this work
Correspondence: Jing-ping Yun Department of Pathology, Sun Yat-sen University Cancer Center, 65I Dongfeng Rd, Guangzhou 510060, People's Republic of China

Tel/fax +86 2087342272

Email yunjplab@163.com
Objective: Hepatocellular carcinoma (HCC) recurrence is a clinical challenge. An accurate prediction system for patients with HCC is needed, since the choice of HCC treatment strategies is very important.

Patients and methods: A total of 804 patients with HCC who underwent curative resection at Sun Yat-sen University Cancer Center were included in this study. Demographics, clinicopathological data, and follow-up information were collected.

Results: A logistic regression analysis was conducted to investigate the relationships between clinical features and $\mathrm{HCC}$ recurrence. Tumor size (OR=1.454, 95\% CI: $1.047-2.020, P=0.026)$ and TNM stage $(\mathrm{OR}=1.360,95 \% \mathrm{CI}: 1.021-1.813, P=0.036)$ were independent predictors of HCC recurrence after curative resection. Therefore, the following equation was established to predict HCC recurrence: $0.308 \times \mathrm{TNM}+0.374 \times$ tumor size -0.639 . The equation score was $0.53 \pm 0.23$ in patients who experienced HCC recurrence compared with $0.47 \pm 0.24$ in other patients. A similar trend was observed in patients who survived after the last follow-up, compared with those who did not, with scores of $0.37 \pm 0.26$ vs $0.52 \pm 0.22$, respectively $(P<0.001)$. The Kaplan-Meier analysis showed that patients with $\mathrm{HCC}$ with equation values $>0.5$ had significantly worse outcomes than those with equation values $\leq 0.5(P<0.001)$ for overall survival $(\mathrm{OS})$ and recurrence $(P=0.043)$. Multivariate Cox analyses showed that tumor multiplicity $(P=0.039)$, involucrum $(P=0.029)$, vascular invasion $(P<0.001)$, and equation value $(P<0.001)$ were independent prognostic variables for OS, whereas tumor multiplicity $(P=0.01)$, tumor differentiation $(P=0.007)$, vascular invasion $(P<0.001)$, involucrum $(P=0.01)$, and equation value $(P<0.001)$ were independent prognostic variables for HCC recurrence.

Conclusion: We established a novel and effective equation for predicting the probability of recurrence and OS after curative resection. Patients with a high recurrence score, based on this equation, should undergo additional high-end imaging examinations.

Keywords: hepatocellular carcinoma, recurrence, tumor size, TNM stage, equation

\section{Introduction}

Hepatocellular carcinoma $(\mathrm{HCC})$ remains the third most common malignancy in the world due to the increased incidence of nonalcoholic fatty liver disease and the high infection rate of hepatitis virus. The prognosis of HCC depends on tumor expansion. ${ }^{1}$ One of the few opportunities for patients with $\mathrm{HCC}$ to achieve a cure is surgical resection. ${ }^{2,3}$ However, it is well known that HCC recurrence after hepatectomy is a major risk factor that affects survival. ${ }^{4}$ A proportion of patients with $\mathrm{HCC}$ experience $\mathrm{HCC}$ 
recurrence after complete $\mathrm{HCC}$ resection. ${ }^{4-6}$ Therefore, $\mathrm{HCC}$ recurrence is a main clinical challenge of HCC treatment. An accurate prediction system for patients with HCC is needed, since the choice of HCC treatment strategy is very important.

Identifying patients with high or low risks of recurrence after hepatectomy for HCC will help determine other therapy and management strategies. ${ }^{7,8}$ Recently, several prognostic staging systems have been reported, such as the Japanese General Stage score, the cancer of the liver Italian program (CLIP) score, and the Barcelona Clinical Liver Cancer staging system. ${ }^{9-12}$ Although these staging systems help divide patients into different groups with different outcomes, they are not suitable for use in predicting recurrence after HCC resection. Therefore, an accurate model is needed to predict the likelihood of HCC recurrence after curative resection.

Although some clinicopathological data, such as tumor multiplicity and serum $\alpha$-fetoprotein (AFP) levels, have been established as poor prognostic indicators and risk factors for HCC recurrence, ${ }^{13-16}$ such clinicopathological data have limited prognostic value when used alone. Combining indicators provides an effective method for improving the prognostic value. Therefore, the objective of this study was to construct an equation for distinguishing the risk of recurrence based on routine markers in patients with $\mathrm{HCC}$ who had undergone an HCC curative resection.

\section{Patients and methods}

\section{Patients}

A total of 804 patients with HCC who underwent curative resection at the Sun Yat-sen University Cancer Center were included in this study. The inclusion criteria were as follows: 1) pathological diagnosis of HCC (by an experienced pathologist), 2) patients with complete clinicopathological and follow-up data, and 3) patients who did not receive any chemotherapy or radiotherapy prior to the surgery. The study protocol was approved by the Clinical Research Ethics Committee of the Sun Yat-sen University Cancer Center. All procedures were followed in accordance with the ethical standards of the responsible committee on human experimentation and with the Declaration of Helsinki (1975), as revised in 2008. Written informed consent was obtained from all patients prior to inclusion in this study.

\section{Demographic and clinicopathological data collection}

Demographic and clinicopathological data, including age, sex, hepatitis B surface antigen (HBsAg), serum AFP level, liver cirrhosis nodule, tumor size, tumor multiplicity, tumor encapsulation, tumor differentiation, TNM stage, and microvascular invasion, were collected. The TNM stage in this study was defined according to the American Joint Committee on Cancer TNM Staging for Liver Tumors as follows: ${ }^{17}$ primary tumor (T): (TX) Primary tumor cannot be assessed; (T0) no evidence of primary tumor; (T1) solitary tumor without vascular invasion; (T2) solitary tumor with vascular invasion or multiple tumors less than $5 \mathrm{~cm}$ in size; (T3a) multiple tumors more than $5 \mathrm{~cm}$ in size; (T3b) single tumor or multiple tumors of any size, involving a major branch of the portal vein or hepatic vein; and (T4) tumor(s) with direct invasion of adjacent organs, other than the gallbladder, or with perforation of visceral peritoneum. Regional lymph nodes $(\mathrm{N})$ : (NX) regional lymph nodes cannot be assessed; (N0) no regional lymph node metastasis and (N1) regional lymph node metastasis. Distant metastasis (M): (M0) no distant metastasis and (M1) distant metastasis.

\section{Follow-up}

After receiving curative hepatectomies, patients with HCC underwent follow-ups and received serological and imaging examinations, including serum AFP level analysis, abdomen ultrasonography, computed tomography (CT), and magnetic resonance imaging (MRI), once every 1-6 months. For patients without evidence of an event, the last follow-up date was obtained from the medical record.

\section{Statistical analyses}

Statistical analyses were performed using SPSS (version 16.0, SPSS Inc., Chicago, IL, USA). A Student's $t$-test and Pearson's chi-squared test, or Fisher's exact test, were chosen for examining the correlations between HCC recurrence and clinical and pathological variables. Survival curves were constructed using the Kaplan-Meier method (log-rank test). A logistic regression was performed to construct the recurrence prediction equation. A multivariate Cox proportional hazards regression model was used to evaluate the independence of the equation in predicting outcomes. Differences with $P$-values less than 0.05 were defined as significant.

\section{Results \\ Associations between clinical features and $\mathrm{HCC}$ recurrence}

The associations between HCC recurrence and clinical features are shown in Table 1 . Significantly more patients experienced HCC recurrences among patients with larger tumor size $(P=0.008)$, poor-undifferentiated tumor differentiation 
Table I Association between clinical features and hepatocellular carcinoma recurrence

\begin{tabular}{|c|c|c|c|}
\hline \multirow[t]{2}{*}{ Variables } & \multicolumn{2}{|l|}{ Recurrence } & \multirow[t]{2}{*}{$P$-value } \\
\hline & Positive & Negative & \\
\hline Sample size & 443 & 361 & \\
\hline Age (years) & $49.21 \pm 11.87$ & $48.45 \pm 12.01$ & 0.369 \\
\hline Sex, n (\%) & & & 0.292 \\
\hline Male & $387(87.4)$ & $324(89.8)$ & \\
\hline Female & $56(12.6)$ & $37(10.2)$ & \\
\hline HBsAg, n (\%) & & & 0.975 \\
\hline Positive & $369(83.3)$ & $301(83.4)$ & \\
\hline Negative & $74(16.7)$ & $60(16.6)$ & \\
\hline AFP (ng/mL), n (\%) & & & 0.949 \\
\hline$<20$ & $99(22.3)$ & $80(22.2)$ & \\
\hline$\geq 20$ & $344(77.7)$ & $281(77.8)$ & \\
\hline Cirrhosis, n (\%) & & & 0.918 \\
\hline Yes & $360(8 I .4)$ & $293(81.2)$ & \\
\hline No & $82(18.6)$ & $68(18.8)$ & \\
\hline Tumor size (cm), n (\%) & & & 0.008 \\
\hline$<5$ & $93(21.0)$ & $105(29.1)$ & \\
\hline$\geq 5$ & $350(79.0)$ & $256(70.9)$ & \\
\hline Tumor multiplicity, n (\%) & & & 0.171 \\
\hline Single & $284(64.1)$ & $248(68.7)$ & \\
\hline Multiple & $159(35.9)$ & $113(31.3)$ & \\
\hline Differentiation, n (\%) & & & 0.042 \\
\hline Well-Moderate & $30(6.8)$ & $39(10.8)$ & \\
\hline Poor-undifferentiated & $413(93.2)$ & $322(89.2)$ & \\
\hline TNM stage, n (\%) & & & 0.011 \\
\hline I-II & $167(37.7)$ & $168(46.5)$ & \\
\hline III-IV & $276(62.3)$ & $193(53.5)$ & \\
\hline Vascular invasion, n (\%) & & & 0.145 \\
\hline Yes & $90(20.4)$ & $59(16.3)$ & \\
\hline No & $352(79.6)$ & $302(83.7)$ & \\
\hline Involucrum, n (\%) & & & 0.108 \\
\hline Complete & $174(39.4)$ & $162(45.0)$ & \\
\hline Incomplete & $268(60.6)$ & $198(55.0)$ & \\
\hline Lymph node metastasis, $\mathrm{n}(\%)$ & & & 0.026 \\
\hline Positive & $32(7.2)$ & $13(3.6)$ & \\
\hline Negative & $410(92.8)$ & $348(96.4)$ & \\
\hline
\end{tabular}

Abbreviations: AFP, $\alpha$-fetoprotein; $\mathrm{HBs} A g$, hepatitis $B$ virus surface antigen.
( $P=0.042)$, III-IV tumor TNM stage $(P=0.011)$, and positive lymph node metastasis $(P=0.026)$.

\section{Construction and performance of the $\mathrm{HCC}$ recurrence equation}

A logistic regression was conducted to analyze the relationships between clinical features and HCC recurrence (Table 2). Tumor size (OR=1.544, 95\% CI: 1.118-2.131, $P=0.008)$, tumor differentiation ( $\mathrm{OR}=1.667,95 \% \mathrm{CI}: 1.014-2.743$, $P=0.044)$, and TNM stage (OR=1.439, 95\% CI: $1.085-1.908$, $P=0.012$ ) were predictors of $\mathrm{HCC}$ recurrence after curative resection. However, only tumor size $(\mathrm{OR}=1.454,95 \% \mathrm{CI}$ : 1.047-2.020, $P=0.026)$ and TNM stage $(\mathrm{OR}=1.360,95 \%$ CI: $1.021-1.813, P=0.036)$ were independent predictors of HCC recurrence after curative resection. Therefore, the following equation was established to predict $\mathrm{HCC}$ recurrence: $0.308 \times \mathrm{TNM}+0.374 \times$ tumor size -0.639 . To validate the equation in the prediction of $\mathrm{HCC}$ recurrence after curative resection, we compared the equation value among patients with different prognoses, as shown in Figure 1. The equation score was $0.53 \pm 0.23$ in patients who experienced an HCC recurrence, compared with $0.47 \pm 0.24$ for other patients. Moreover, a similar trend was observed in patients who survived after the last follow-up, compared with those who $\operatorname{did}$ not $(0.37 \pm 0.26$ vs $0.52 \pm 0.22[P<0.001])$.

\section{Performance of the HCC recurrence equation in patient prognosis}

To determine the prognostic impact of the equation on patients with HCC, we conducted a Kaplan-Meier survival analysis using data from the 804 patients with $\mathrm{HCC}$ who were enrolled in this study. Based on the mean equation values,

Table 2 Logistic regression of prognostic variables for hepatocellular carcinoma recurrence

\begin{tabular}{|c|c|c|c|c|c|c|}
\hline \multirow[t]{2}{*}{ Variables } & \multicolumn{3}{|c|}{ Univariate analysis } & \multicolumn{3}{|c|}{ Multivariate analysis } \\
\hline & OR & $95 \% \mathrm{Cl}$ & $P$ & OR & $95 \% \mathrm{Cl}$ & $P$ \\
\hline Age (years) & $\mathrm{I} .005$ & $0.994-1.017$ & 0.369 & & & \\
\hline Sex & $\mathrm{I} .267$ & $0.816-1.969$ & 0.292 & & & \\
\hline $\mathrm{HBsAg}$ & 0.994 & $0.685-1.443$ & 0.975 & & & \\
\hline AFP & 0.989 & $0.708-1.382$ & 0.949 & & & \\
\hline Cirrhosis & 1.019 & $0.713-1.455$ & 0.918 & & & \\
\hline Tumor size $(\mathrm{cm})$ & $\mathrm{I} .544$ & $1.118-2.131$ & 0.008 & 1.454 & $1.047-2.020$ & 0.026 \\
\hline Tumor multiplicity & 1.229 & $0.915-1.651$ & 0.172 & & & \\
\hline Differentiation & 1.667 & $1.014-2.743$ & 0.044 & & & \\
\hline TNM & 1.439 & $1.085-1.908$ & 0.012 & 1.360 & $1.021-1.813$ & 0.036 \\
\hline Vascular invasion & 1.309 & $0.911-1.881$ & 0.146 & & & \\
\hline Involucrum & 1.260 & $0.95 I-|.67|$ & 0.108 & & & \\
\hline
\end{tabular}

Abbreviations: AFP, $\alpha$-fetoprotein; $\mathrm{HBsAg}$, hepatitis $B$ virus surface antigen. 

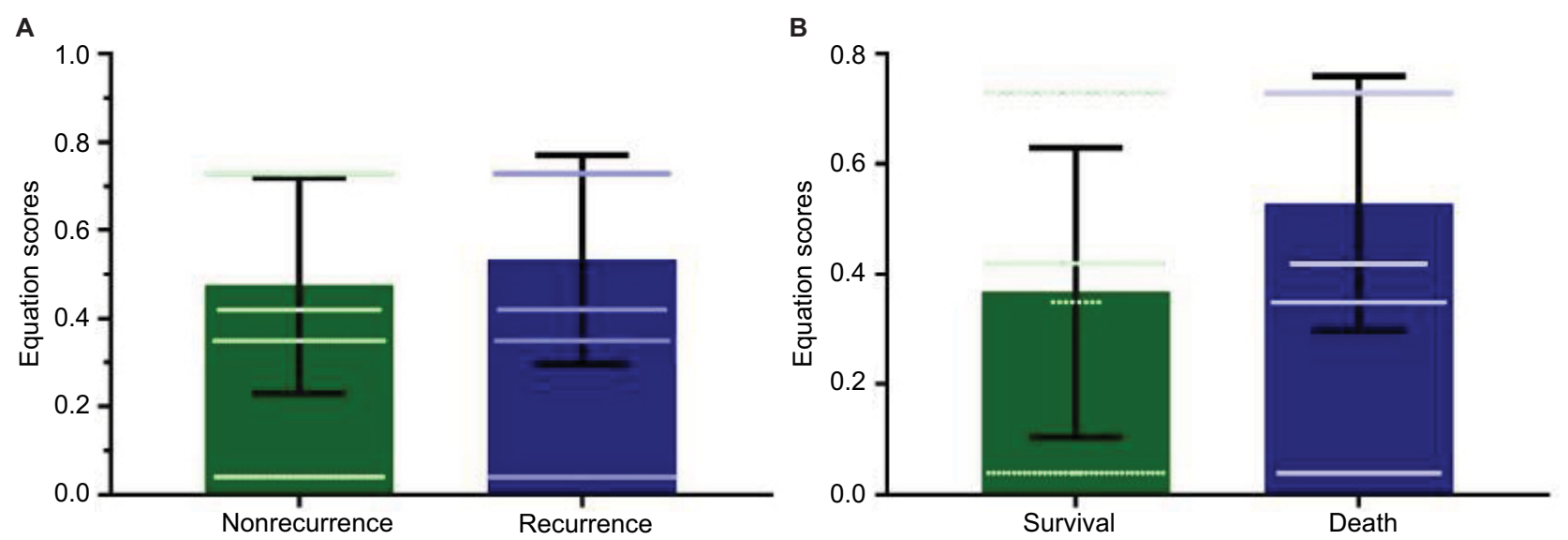

Figure I Equation scores in patients with different prognoses.

Notes: $(\mathbf{A})$ The equation score was significantly higher for patients who experienced hepatocellular carcinoma recurrence than for patients without recurrence $(0.53 \pm 0.23$ vs $0.47 \pm 0.24, P=0.001$ ). (B) Similarly, the equation score was $0.37 \pm 0.26$ in patients who achieved survival, which was significantly lower than that of patients who did not, $0.53 \pm 0.23$.

A

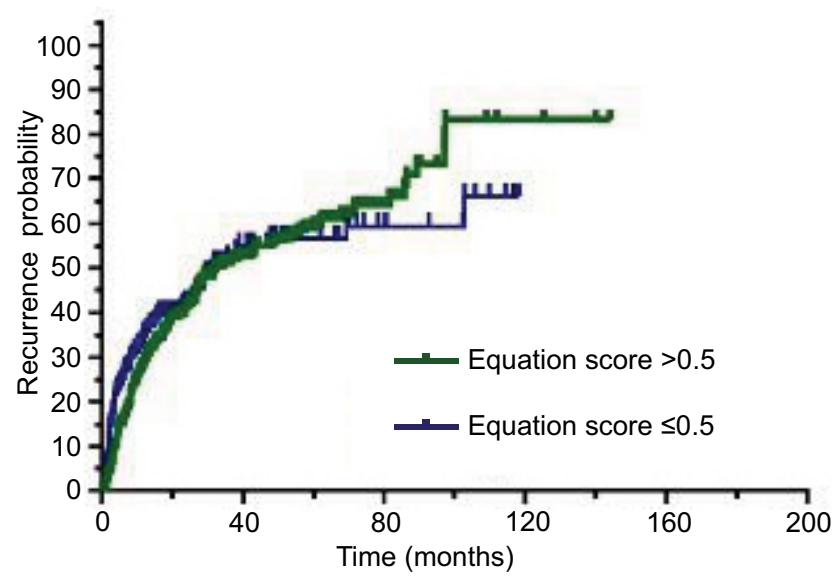

B

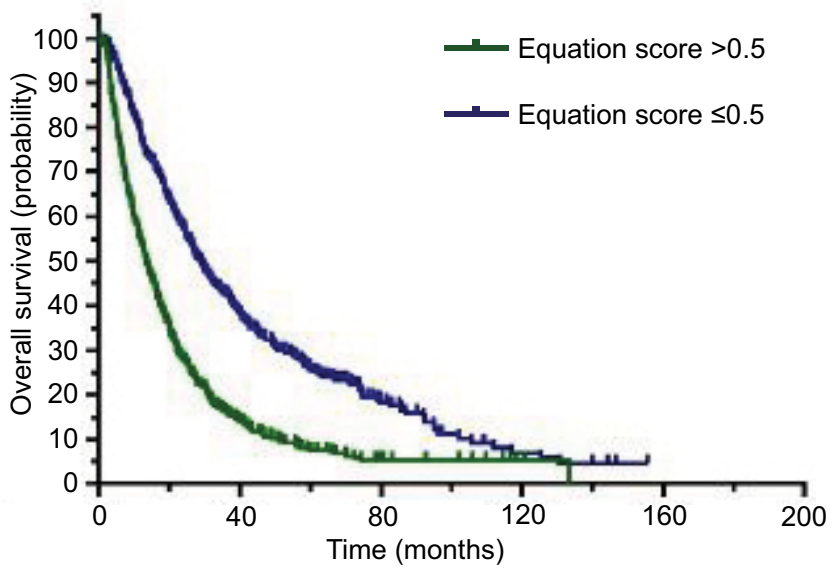

Figure 2 Higher equation score correlates with an unfavorable prognosis for patients with HCC.

Notes: $(\mathbf{A})$ The Kaplan-Meier analysis showed significant differences in recurrence probabilities between $\mathrm{HCC}$ patients with equation scores $>0.5$ and $\leq 0.5$ ( $P=0.043$ ). (B) In addition, a significant difference was observed in overall survival for patients with $\mathrm{HCC}$ with equation scores $>0.5$ and $\leq 0.5$ ( $P<0.00$ I).

Abbreviation: HCC, hepatocellular carcinoma.

we divided the 804 patients into two groups: those with equation values $>0.5$ and those with equation values $\leq 0.5$. In the equation value $>0.5$ cohort, the Kaplan-Meier analysis revealed that these patients with $\mathrm{HCC}$ had significantly worse recurrence outcomes than those in the other cohort $(P=0.043)$. Similar trends were observed for overall survival (OS), which showed that patients with $\mathrm{HCC}$ with equation values $>0.5$ had significantly worse outcomes than those with equation values $\leq 0.5(P<0.001)$, as shown in Figure 2 .

To further explore the relationship between the equation and clinical features, patients with HCC with equation values $>0.5$ were compared to those with equation values $\leq 0.5$ (Table 3). The patients with HCC with equation values $>0.5$ exhibited the majority of poor HCC clinical features.

\section{Univariate and multivariate Cox analyses of HCC prognostic variables}

To evaluate whether equation value was an independent risk factor for HCC outcomes, both univariate and multivariate Cox analyses were conducted. Age, serum AFP level, tumor size, tumor multiplicity, tumor differentiation, TNM stage, vascular invasion, involucrum, and equation value were all found to be prognostic variables for OS in patients with HCC. In the multivariate analysis, only tumor multiplicity $(P=0.039)$, involucrum $(P=0.029)$, vascular invasion $(P<0.001)$, and equation value $(P<0.001)$ were independent prognostic variables that were associated with OS (Table 4). Similarly, after conducting univariate and multivariate Cox analyses, tumor multiplicity $(P=0.01)$, tumor differentiation 
( $P=0.007)$, vascular invasion $(P<0.001)$, involucrum $(P=0.01)$, and equation value $(P<0.001)$ were found to be independent prognostic variables for HCC recurrence (Table 5).

Table 3 Association between clinical features and equation scores in hepatocellular carcinoma

\begin{tabular}{|c|c|c|c|}
\hline \multirow[t]{2}{*}{ Variables } & \multicolumn{2}{|c|}{ Equation score } & \multirow[t]{2}{*}{$P$-value } \\
\hline & $>0.5$ & $\leq 0.5$ & \\
\hline Sample size & 382 & 422 & \\
\hline Age (years) & $47.95 \pm 12.12$ & $49.71 \pm 11.72$ & 0.036 \\
\hline Sex, n (\%) & & & 0.355 \\
\hline Male & $342(89.5)$ & $369(87.4)$ & \\
\hline Female & $40(10.5)$ & $53(12.6)$ & \\
\hline HBsAg, n (\%) & & & 0.016 \\
\hline Positive & $331(86.6)$ & $339(80.3)$ & \\
\hline Negative & $51(13.4)$ & $83(19.7)$ & \\
\hline AFP (ng/mL), n (\%) & & & $<0.00$ I \\
\hline$<20$ & $60(15.7)$ & $119(28.2)$ & \\
\hline$\geq 20$ & $322(84.3)$ & 303 (7I.8) & \\
\hline Cirrhosis, n (\%) & & & $<0.00$ I \\
\hline Yes & $289(75.7)$ & 364 (86.5) & \\
\hline No & $93(24.3)$ & $57(13.5)$ & \\
\hline Tumor multiplicity, n (\%) & & & $<0.001$ \\
\hline Single & I 75 (45.8) & $357(84.6)$ & \\
\hline Multiple & $207(54.2)$ & $65(15.4)$ & \\
\hline Differentiation, n (\%) & & & $<0.001$ \\
\hline Well-moderate & $14(3.7)$ & $55(13.0)$ & \\
\hline Poor-undifferentiated & $368(96.3)$ & $367(87.0)$ & \\
\hline Vascular invasion, n (\%) & & & $<0.001$ \\
\hline Yes & $125(32.7)$ & $24(5.7)$ & \\
\hline No & $257(67.3)$ & 397 (94.3) & \\
\hline Involucrum, n (\%) & & & 0.051 \\
\hline Complete & I46 (38.3) & $190(45.1)$ & \\
\hline Incomplete & $235(61.7)$ & 231 (54.9) & \\
\hline Lymph node metastasis, n (\%) & & & 0.020 \\
\hline Positive & $29(7.6)$ & $16(3.8)$ & \\
\hline Negative & $353(92.4)$ & $405(96.2)$ & \\
\hline
\end{tabular}

Abbreviations: AFP, $\alpha$-fetoprotein; $\mathrm{HBsAg}$, hepatitis $B$ virus surface antigen.

\section{Subgroup analyses of equation values in patients with $\mathrm{HCC}$}

A stratified survival analysis was also conducted to further reveal the significance of equation values among patients with HCC. The equation score was significantly higher in patients with multiple tumors than in those with single tumors $(0.63 \pm 0.17$ vs $0.44 \pm 0.24, P<0.001)$. Additionally, the equation score in patients with incomplete involucrum was $0.52 \pm 0.23$, compared to $0.48 \pm 0.24$ in patients with complete involucrum $(P=0.042)$. The equation score was $0.52 \pm 0.23$ and $0.44 \pm 0.24$, respectively, in patients with abnormal and normal serum AFP levels $(P<0.001)$. Among patients with well-differentiated tumors, the equation score was $0.35 \pm 0.25$, compared with $0.52 \pm 0.23$ in patients with poorly differentiated tumors $(P<0.001)$. For patients with and without liver cirrhosis, the equation scores were $0.49 \pm 0.24$ and $0.57 \pm 0.22$ $(P<0.001)$, respectively, whereas in patients with positive and negative vascular invasion, the scores were $0.67 \pm 0.14$ and $0.47 \pm 0.24$, respectively $(P<0.001)$, as shown in Figure 3 .

\section{Discussion}

In this study, we explored the risk factors for recurrence in patients with $\mathrm{HCC}$ who underwent curative resection. Moreover, we established a novel, effective, and valid equation for predicting the probability of recurrence and OS after curative resection. TNM stage and tumor size were integrated into the equation, and the Kaplan-Meier survival analysis showed that patients with $\mathrm{HCC}$ with higher equation values displayed worse outcomes.

The etiology of HCC is variable and includes chronic virus infection, nonalcoholic liver disease, aflatoxin, and other complications. ${ }^{18}$ Due to the increased incidence of

Table 4 Univariate and multivariate analyses of prognostic variables for overall survival

\begin{tabular}{|c|c|c|c|c|c|c|}
\hline \multirow[t]{2}{*}{ Variables } & \multicolumn{3}{|c|}{ Univariate analysis } & \multicolumn{3}{|c|}{ Multivariate analysis } \\
\hline & OR & $95 \% \mathrm{Cl}$ & $P$ & OR & $95 \% \mathrm{Cl}$ & $P$ \\
\hline Age (years) & 0.991 & $0.984-0.997$ & 0.004 & & & \\
\hline Sex & 0.870 & $0.683-1.110$ & 0.263 & & & \\
\hline $\mathrm{HBsAg}$ & 1.166 & $0.949-1.433$ & 0.143 & & & \\
\hline AFP & 1.254 & $1.048-1.502$ & 0.014 & & & \\
\hline Cirrhosis & 0.976 & $0.800-1.190$ & 0.808 & & & \\
\hline Tumor size $(\mathrm{cm})$ & 1.644 & $1.370-1.972$ & $<0.001$ & & & \\
\hline Tumor multiplicity & 1.640 & $1.400-1.921$ & $<0.001$ & 1.199 & $1.010-1.424$ & 0.039 \\
\hline Differentiation & 1.627 & $1.239-2.136$ & $<0.001$ & & & \\
\hline TNM & 2.078 & $1.774-2.433$ & $<0.001$ & & & \\
\hline Vascular invasion & 2.586 & $2.139-3.126$ & $<0.001$ & 1.891 & $1.54 \mid-2.320$ & $<0.001$ \\
\hline Involucrum & 1.376 & $1.178-1.607$ & $<0.001$ & 1.196 & $1.018-1.404$ & 0.029 \\
\hline Equation score & 4.952 & $3.535-6.937$ & $<0.001$ & 3.242 & $2.23|-4.7| I$ & $<0.001$ \\
\hline
\end{tabular}

Abbreviations: AFP, $\alpha$-fetoprotein; $\mathrm{HBsAg}$, hepatitis $B$ virus surface antigen. 
Table 5 Univariate and multivariate analyses of prognostic variables for recurrence

\begin{tabular}{|c|c|c|c|c|c|c|}
\hline \multirow[t]{2}{*}{ Variables } & \multicolumn{3}{|c|}{ Univariate analysis } & \multicolumn{3}{|c|}{ Multivariate analysis } \\
\hline & OR & $95 \% \mathrm{Cl}$ & $P$ & OR & $95 \% \mathrm{Cl}$ & $P$ \\
\hline Age (years) & 0.991 & $0.983-0.999$ & 0.022 & & & \\
\hline Sex & 0.983 & $0.738-1.309$ & 0.905 & & & \\
\hline $\mathrm{HBsAg}$ & 1.138 & $0.88 \mathrm{I}-1.470$ & 0.322 & & & \\
\hline AFP & 1.293 & $1.032-1.62 \mid$ & 0.026 & & & \\
\hline Cirrhosis & 0.990 & $0.776-1.263$ & 0.935 & & & \\
\hline Tumor size $(\mathrm{cm})$ & 1.800 & $1.427-2.269$ & $<0.001$ & & & \\
\hline Tumor multiplicity & 1.837 & $1.503-2.245$ & $<0.001$ & 1.329 & $1.070-1.650$ & 0.010 \\
\hline Differentiation & 2.216 & $1.528-3.215$ & $<0.001$ & 1.695 & I.152-2.493 & 0.007 \\
\hline TNM & 2.153 & $1.765-2.625$ & $<0.001$ & & & \\
\hline Vascular invasion & 2.750 & $2.155-3.508$ & $<0.001$ & 1.806 & $|.388-2.35|$ & $<0.001$ \\
\hline Involucrum & 1.543 & $1.269-1.878$ & $<0.001$ & 1.312 & $1.068-1.611$ & 0.010 \\
\hline Equation score & 5.474 & $3.602-8.320$ & $<0.001$ & 3.110 & $1.965-4.922$ & $<0.001$ \\
\hline
\end{tabular}

Abbreviations: AFP, $\alpha$-fetoprotein; $\mathrm{HBsAg}$, hepatitis B virus surface antigen.

A

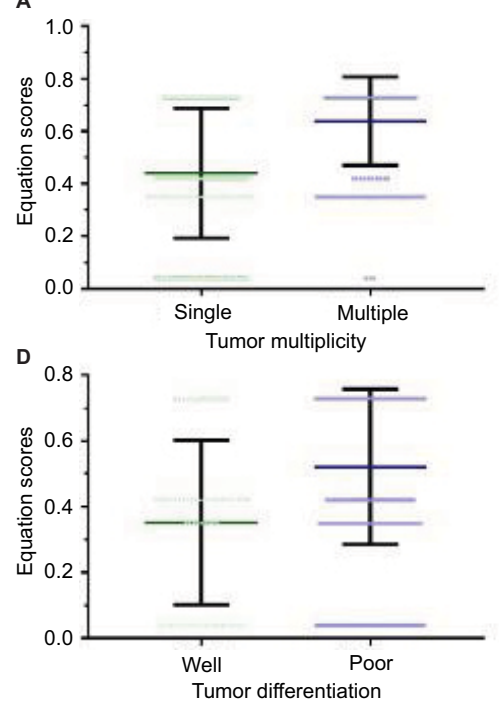

B
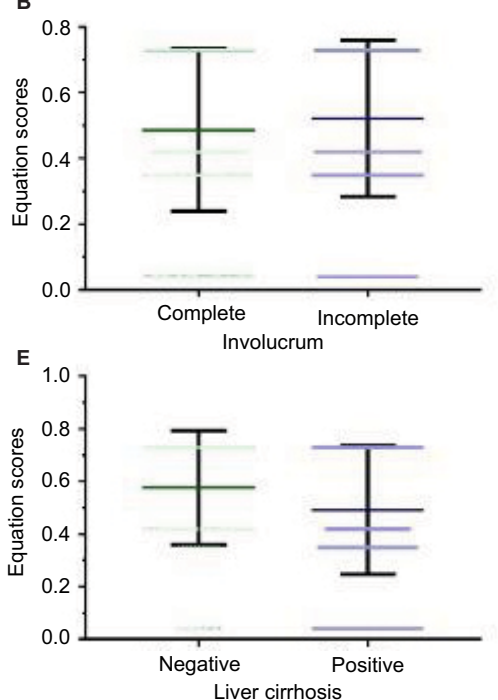

C

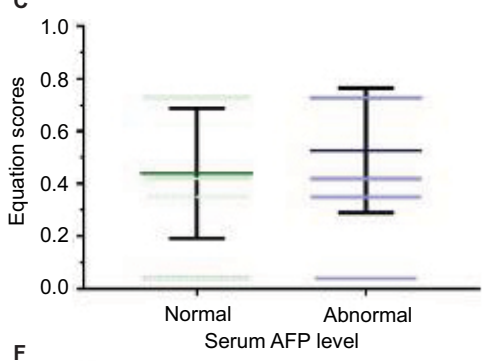

F

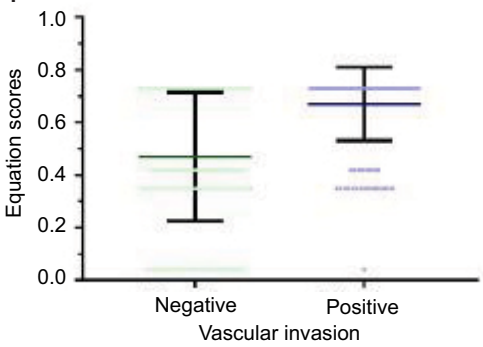

Figure 3 Equation scores in HCC subpopulations.

Notes: (A) The equation score was significantly higher in patients with multiple tumors than in those with a single tumor $(0.63 \pm 0.17$ vs $0.44 \pm 0.24, P<0.001)$. (B) In patients with incomplete involucrum, the equation value was $0.52 \pm 0.23$, compared to $0.48 \pm 0.24$ in patients with complete involucrum ( $P=0.042$ ). (C) The equation score was $0.52 \pm 0.23$ and $0.44 \pm 0.24$, respectively, in patients with abnormal and normal serum AFP levels $(P<0.00 \mathrm{I})$. (D) Among patients with well-differentiated tumors, the equation score was $0.35 \pm 0.25$, compared to $0.52 \pm 0.23$ in patients with poorly differentiated tumors $(P<0.00 \mathrm{I})$. (E) For patients with and without liver cirrhosis, the equation scores were $0.49 \pm 0.24$ and $0.57 \pm 0.22(P<0.001)$, respectively, $(\mathbf{F})$ whereas in patients with positive and negative vascular invasion, the scores were $0.67 \pm 0.14$ and $0.47 \pm 0.24$, respectively $(P<0.00 \mathrm{I})$.

Abbreviations: AFP, $\alpha$-fetoprotein; HCC, hepatocellular carcinoma.

obesity in the Western countries and the chronic virus infections in Asia, the incidence of HCC has remained high. ${ }^{19-21}$ Many studies have demonstrated the prognostic value of TNM stage, tumor burden, and impaired liver function (liver fibrosis, liver cirrhosis, and decompensated liver function) in HCC. ${ }^{22-27}$ Other studies have also reported that for HCC caused by chronic hepatitis B virus infection, many HBVrelated indicators, including hepatitis B virus DNA load, HBsAg, and antiviral treatment, also affect the prognosis of patients with HCC. ${ }^{21,28-33}$ However, these prognostic indicators have limited value when used alone. How to combine data to improve prognostic value is a clinically practical issue. The CLIP score was the first system that took liver function and tumor characteristics into consideration for the classification of HCC treatment. However, the prognostic performance was reported to be poor because $\sim 80 \%$ of the patients were classified with scores of $0-2 .{ }^{34,35}$ In this study, we combined TNM stage and tumor size into an equation. We have conducted a multivariable logistic regression analysis and included age, sex, HBsAg status, AFP level, 
tumor multiplicity, and other variables to determine which variables are most related to $\mathrm{HCC}$ recurrence and constructed a prediction equation. Our results suggest that TNM stage and tumor size are most relevant to $\mathrm{HCC}$ recurrence. Therefore, other variables were not included in our equation, and the Kaplan-Meier survival analysis shows that the equation value could effectively predict the outcomes of the HCC population.

Combining several markers into one equation allows an analysis of patients with HCC with more comprehensive information and provides individualized risk assessments. Similar to the results from a previous study, our results indicate that tumor size is an independent risk factor for recurrence in patients with HCC. ${ }^{36}$ Tumor size is one of the most important tumor burden parameters. The results of this and previous studies indicate that advanced TNM stage closely associates with the development of HCC because of its positive correlation with tumor size and poor OS. ${ }^{37,38}$ Our equation exhibited superior discrimination of $\mathrm{HCC}$ recurrence in patients. Hence, the equation could be used to guide routine follow-up for patients. Especially, patients with HCC with high recurrence scores should undergo examinations more frequently, such as MRI or CT examinations, even if the most recent examination after curative resection indicates no cause for concern.

AFP and tumor multiplicity have been reported as prognostic markers in HCC. ${ }^{39}$ AFP is a serum HCC marker that has been previously used to monitor HCC recurrence. However, AFP levels can also be elevated in some diseases, such as liver cirrhosis and female reproductive system tumors. ${ }^{40,41}$ Although high preoperative AFP levels are associated with poorer $\mathrm{HCC}$ outcomes, there is a certain proportion of patients with HCC with AFP levels that are within the upper limit of normal. ${ }^{42}$ For those AFP-negative patients with HCC, AFP is not a suitable prognostic marker. In this study, patients with HCC with abnormal or normal AFP levels could be effectively stratified using this equation. Tumor multiplicity is another prognostic marker that has been reported in HCC. ${ }^{43}$ According to this study, equation scores significantly differ between patients with a single HCC and those with multiple HCCs. No matter whether patients with HCC have a single tumor or multiple tumors, our equation can further risk stratify these subpopulations with HCC.

Whether or not the equation can improve the prognosis of patients with HCC remains an interesting and important question. The main reason why HCC is difficult to treat is its high recurrence rate. Moreover, the clinical symptoms of HCC are not obvious. When the typical symptoms occur, HCC has typically progressed to a state that makes treatment difficult. Therefore, the method for screening patients with $\mathrm{HCC}$ after surgery to facilitate early recurrence detection is a clinically critical problem. The early detection of HCC recurrence and early intervention can improve patient prognosis. However, high-frequency screening of all patients is not a cost-effective strategy. According to the results of this study, high-frequency follow-up and screening for high-risk patients, early detection of $\mathrm{HCC}$ recurrence, and early interventions may ultimately improve the prognosis of patients. However, this requires further prospective studies to confirm.

\section{Conclusion}

Here we established a novel and effective equation for predicting the probability of recurrence and OS after curative resection. Patients with a high recurrence score, based on this equation, should undergo additional high-end imaging examinations. Although our equation showed good performance, several limitations need to be addressed. First, the equation was derived from data collected at a single institution. Second, the etiology of HCC in China is mostly due to chronic hepatitis B. Third, because this study was a retrospective study, the results may be biased. For future performance algorithms, prospective cohort studies are needed.

\section{Acknowledgment}

This study was supported by the Guangdong Provincial Medical Research Fund (A2018003).

\section{Disclosure}

The authors declare that they have no financial or personal relationships with other people or organizations that could inappropriately influence this work. The authors report no other conflicts of interest in this work.

\section{References}

1. Forner A, Reig M, Bruix J. Hepatocellular carcinoma. Lancet. 2018;391(10127):1301-1314.

2. Granito A, Bolondi L. Non-transplant therapies for patients with hepatocellular carcinoma and Child-Pugh-Turcotte class B cirrhosis. Lancet Oncol. 2017;18(2):e101-e112.

3. Cai SH, Lu SX, Liu LL, Zhang CZ, Yun JP. Increased expression of hepatocyte nuclear factor 4 alpha transcribed by promoter 2 indicates a poor prognosis in hepatocellular carcinoma. Therap Adv Gastroenterol. 2017;10(10):761-771.

4. Chang K-V, Chen J-D, Wu W-T, Huang K-C, Hsu C-T, Han D-S. Association between loss of skeletal muscle mass and mortality and tumor recurrence in hepatocellular carcinoma: a systematic review and meta-analysis. Liver Cancer. 2018;7(1):90-103.

5. Forner A, Llovet JM, Bruix J. Hepatocellular carcinoma. Lancet 2012;379(9822):1245-1255.

6. Hirokawa F, Hayashi M, Miyamoto Y, et al. Outcomes and predictors of microvascular invasion of solitary hepatocellular carcinoma. Hepatol Res. 2014;44(8):846-853. 
7. Xiao Y, Li W, Wan H, Tan Y, Wu H. Central hepatectomy versus major hepatectomy for patients with centrally located hepatocellular carcinoma: a meta-analysis. Int J Surg. 2018;52:297-302.

8. Butt AS, Sharif F, Abid S. Impact of direct acting antivirals on occurrence and recurrence of hepatocellular carcinoma: biologically plausible or an epiphenomenon? World J Hepatol. 2018;10(2):267-276.

9. Toyoda H, Hiraoka A, Tada T, et al. Characteristics and prognosis of hepatocellular carcinoma in Japanese patients undergoing dialysis. Ther Apher Dial. 2017;21(5):465-472.

10. Sato T, Takahashi Y, Imai M, Isokawa O. Hepatic arterial infusion chemotherapy using a reservoir for advanced hepatocellular carcinoma. Gan To Kagaku Ryoho. 2016;43(1):73-77.

11. Borzio M, Dionigi E, Rossini A, et al. External validation of the ITA. LI.CA prognostic system for patients with hepatocellular carcinoma: a multicenter cohort study. Hepatology. 2017.

12. Yang Z, Ye P, Xu Q, et al. Elevation of serum GGT and LDH levels, together with higher BCLC staging are associated with poor overall survival from hepatocellular carcinoma: a retrospective analysis. Discov Med. 2015;19(107):409-418.

13. Yu SJ, Kwon JH, Kim W, et al. Initial alpha-fetoprotein response predicts prognosis in hepatitis B-related solitary HCC patients after radiofrequency ablation. J Clin Gastroenterol. 2018;52(3):e18-e26.

14. Lee K, Lee KB, Yi NJ, Suh KS, Jang JJ. Prognosis of hepatocellular carcinoma after liver transplantation: comparative analysis with partial hepatectomy. J Pathol Transl Med. 2017;51(1):79-86.

15. Koh KC, Lee H, Choi MS, et al. Clinicopathologic features and prognosis of combined hepatocellular cholangiocarcinoma. Am J Surg. 2005;189(1):120-125.

16. Ikeda K, Saitoh S, Tsubota A, et al. Risk factors for tumor recurrence and prognosis after curative resection of hepatocellular carcinoma. Cancer. 1993;71(1):19-25.

17. Edge SB, Compton CC. The American Joint Committee on Cancer: the 7th edition of the AJCC cancer staging manual and the future of TNM. Ann Surg Oncol. 2010;17(6):1471-1474.

18. Cai S, Cao J, Yu T, Xia M, Peng J. Effectiveness of entecavir or telbivudine therapy in patients with chronic hepatitis B virus infection pretreated with interferon compared with de novo therapy with entecavir and telbivudine. Medicine. 2017;96(22):e7021.

19. Cai S, Ou Z, Liu D, et al. Risk factors associated with liver steatosis and fibrosis in chronic hepatitis B patient with component of metabolic syndrome. United European Gastroenterol J. 2018;6(4):558-566.

20. Ou H, Cai S, Liu Y, Xia M, Peng J. A noninvasive diagnostic model to assess nonalcoholic hepatic steatosis in patients with chronic hepatitis B. Therap Adv Gastroenterol. 2017;10(2):207-217.

21. Xue X, Cai S, Ou H, Zheng C, Wu X. Health-related quality of life in patients with chronic hepatitis B during antiviral treatment and offtreatment. Patient Prefer Adherence. 2017;11:85-93.

22. Ho SY, Liu PH, Hsu CY, et al. Prognostic performance of ten liver function models in patients with hepatocellular carcinoma undergoing radiofrequency ablation. Sci Rep. 2018;8(1):843.

23. Na SK, Yim SY, Suh SJ, et al. ALBI versus Child-Pugh grading systems for liver function in patients with hepatocellular carcinoma. $J$ Surg Oncol. 2018;117(5):912-921.

24. Chang KV, Chen JD, Wu WT, Huang KC, Hsu CT, Han DS. Association between loss of skeletal muscle mass and mortality and tumor recurrence in hepatocellular carcinoma: a systematic review and meta-analysis. Liver Cancer. 2018;7(1):90-103.

25. Shen J, Liu J, Li C, Wen T, Yan L, Yang J. The prognostic significance of serum $\mathrm{HBeAg}$ on the recurrence and long-term survival after hepatectomy for hepatocellular carcinoma: a propensity score matching analysis. J Viral Hepat. 2018
26. Komorowski AL, Hsu CC, Julka KD, et al. AFP role in predicting recurrence of hepatocellular carcinoma after living donor liver transplantation in HCV patients. Neoplasma. 2018;65(3):455-460.

27. Xue X, Cai S. Comment on "Assessment of liver stiffness in pediatric fontan patients using transient elastography". Can J Gastroenterol Hepatol. 2016;2016:9343960-2.

28. Huang G, Li PP, Lau WY, et al. Antiviral therapy reduces hepatocellular carcinoma recurrence in patients with low HBV-DNA levels: a randomized controlled trial. Ann Surg. 2018:1.

29. Yang X, Gao JY, Wang J, Cheng J. The impact of anti-HBV treatment on the occurrence and recurrence of hepatocellular carcinoma: focus on Asian studies. Discov Med. 2015;19(103):89-99.

30. Zhang L, Xie XY, Chen Y, et al. Hepatitis B surface antigen predicts recurrence after radiofrequency ablation in patients with low hepatitis B virus loads. Medicine. 2017;96(52):e9377.

31. Zeng J, Cai S, Liu J, Xue X, Wu X, Zheng C. Dynamic changes in liver stiffness measured by transient elastography predict clinical outcomes among patients with chronic hepatitis B. J Ultrasound Med. 2017;36(2): 261-268.

32. Cai S, Yu T, Jiang Y, Zhang Y, Lv F, Peng J. Comparison of entecavir monotherapy and de novo lamivudine and adefovir combination therapy in $\mathrm{HBe} A g$-positive chronic hepatitis B with high viral load: 48-week result. Clin Exp Med. 2016;16(3):429-436.

33. Cai S, Li Z, Yu T, Xia M, Peng J. Serum hepatitis B core antibody levels predict $\mathrm{HBeAg}$ seroconversion in chronic hepatitis B patients with high viral load treated with nucleos(t)ide analogs. Infect Drug Resist. 2018;11:469-477.

34. Gan W, Huang JL, Zhang MX, et al. New nomogram predicts the recurrence of hepatocellular carcinoma in patients with negative preoperative serum AFP subjected to curative resection. J Surg Oncol. 2018;117(7):1540-1547.

35. Tokumitsu Y, Sakamoto K, Tokuhisa Y, et al. A new prognostic model for hepatocellular carcinoma recurrence after curative hepatectomy. Oncol Lett. 2018;15(4):4411-4422.

36. Mcpeake JR, O'Grady JG, Zaman S, et al. Liver transplantation for primary hepatocellular carcinoma: tumor size and number determine outcome. J Hepatol. 1993;18(2):226-234.

37. Gao Q, Wang XY, Qiu SJ, et al. Tumor stroma reaction-related gene signature predicts clinical outcome in human hepatocellular carcinoma. Cancer Sci. 2011;102(8):1522-1531.

38. Zimmerman MA, Trotter JF, Wachs M, et al. Predictors of long-term outcome following liver transplantation for hepatocellular carcinoma: a single-center experience. Transpl Int. 2007;20(9):747-753.

39. Notarpaolo A, Layese R, Magistri P, et al. Validation of the AFP model as a predictor of $\mathrm{HCC}$ recurrence in patients with viral hepatitis-related cirrhosis who had received a liver transplant for HCC. $J$ Hepatol. 2017;66(3):552-559.

40. Li D, Satomura S. Biomarkers for hepatocellular Carcinoma (HCC): an update. Adv Exp Med Biol. 2015;867:179-193.

41. Toyoda H, Kumada T, Tada T, Sone Y, Kaneoka Y, Maeda A. Tumor markers for hepatocellular carcinoma: simple and significant predictors of outcome in patients with HCC. Liver Cancer. 2015;4(2): 126-136.

42. Baig JA, Alam JM, Mahmood SR, et al. Hepatocellular carcinoma (HCC) and diagnostic significance of A-fetoprotein (AFP). JAyub Med Coll Abbottabad. 2009;21(1):72-75.

43. Du ZG, Wei YG, Chen KF, Li B. Risk factors associated with early and late recurrence after curative resection of hepatocellular carcinoma: a single institution's experience with 398 consecutive patients. Hepatobiliary Pancreat Dis Int 2014;13:153-61. 
Cancer Management and Research

\section{Publish your work in this journal}

Cancer Management and Research is an international, peer-reviewed open access journal focusing on cancer research and the optimal use of preventative and integrated treatment interventions to achieve improved outcomes, enhanced survival and quality of life for the cancer patient.

The manuscript management system is completely online and includes

Submit your manuscript here: https://www.dovepress.com/cancer-management-and-research-journal

a very quick and fair peer-review system, which is all easy to use. Visit $\mathrm{http}: / / \mathrm{www}$.dovepress.com/testimonials.php to read real quotes from published authors. 